\title{
Association between arthralgia and imaging findings of effusion in the temporomandibular joints: a systematic review
}

\begin{abstract}
Aims: A systematic review was conducted to determine if there is an association between joint effusion and self-reported TMJ-pain during examination and between joint effusion and arthralgia as defined by the Research Diagnostic Criteria for Temporomandibular Disorders (RDC-TMD)
\end{abstract}

Methods: Four reviewers separately identified observational studies evaluating a possible association between ipsilateral joint effusion, identified by MRI T2-weighted images, and TMJ pain by systematically searching three databases.

Results: A total of 67 articles were identified with the search strategy. However, 32 met the inclusion criteria for the systematic review.

Conclusion: Based on the review of 32 articles, published on this topic, body of literature was unable to provide evidence to support or refute the association between joint effusion and self-reported TMJ-pain, and between joint effusion and arthralgia as defined by RDCTMD.

Keywords: temporomandibular disorders, joint effusion, MRI, arthralgia, pain
Volume 7 Issue 6 - 2017

\author{
Heidi Crow,' Shehryar N Khawaja, ${ }^{2,3,4}$ Ruba \\ FG Mahmoud, ${ }^{4}$ Krishnan Kartha, ${ }^{5}$ Willard \\ McCall, ${ }^{4}$ Yoly Gonzalez ${ }^{4}$ \\ 'Department of Oral Diagnostic Sciences, University at Buffalo, \\ USA \\ 2Department of Oral and Maxillofacial Surgery, Massachusetts \\ General Hospital, USA \\ ${ }^{3}$ Harvard School of Dental Medicine, Harvard University, USA \\ ${ }^{4}$ Department of Oral Diagnostic Sciences, University at Buffalo, \\ USA \\ ${ }^{5}$ Department of TMD and Orofacial Pain, King Fahad Armed \\ Forces Hospital, Saudi Arabia
}

\begin{abstract}
Correspondence: Shehryar N Khawaja, Department of Ora and Maxillofacial Surgery, Massachusetts General Hospital, 55 Fruit Street, Warren I201, Boston, Massachusetts, 02 I I4, USA, Tel I4357I0 I080, Fax I7I68293554, Email khawajashehryar@gmail.com
\end{abstract}

Received: March 27, 2017 | Published: August 02, 2017
Abbreviations: MRI, magnetic resonance imaging; $\mathrm{JE}$, joint effusion; RDC/TMD, research diagnostic criteria for temporomandibular disorders; VGIR, visually guided TMJ irrigation; ID, internal derangement; DJD, degenerative joint disease; DDwR, disk displacement with reduction; DDwoR, disk displacement without reduction; OA, osteoarthrosis; CDC/TMD, clinical diagnostic criteria for temporomandibular disorders; OR, odds ratio; VAS, visual analogue scale; ICC, interclass correlation coefficients; SSI, symptom severity index

\section{Introduction}

The temporomandibular disorders (TMD) encompass a group of musculoskeletal and neuromuscular conditions that involve the temporomandibular joints (TMJs), the masticatory muscles, and associated tissues. ${ }^{1}$ Common manifestations of TMD consist of pain of a persistent, recurring, or chronic nature in the TMJ, masticatory muscles, or in the adjacent structures; limitation or other alterations in the range of mandible motion; and TM joint noises. ${ }^{2}$ It has been suggested that differential diagnosis of TMDs should be based primarily on information obtained from the patient's history, clinical examination, and when TMJ imaging procedures indicated. ${ }^{1}$ Magnetic resonance imaging (MRI) is an example of such imaging procedures. It provides excellent representation of soft tissues in anatomical and semi-functional relationships. In addition, it may also be used for detection of the presence of joint effusion with acceptable levels of reliability. ${ }^{3}$ Some authors have also suggested use of MRI for assessment of hard tissues; however the reliability has been shown to be poor. ${ }^{3-5}$ Joint effusion is defined as a collection of fluid in the joint space. It is manifested as areas of high intensity signal in the TMJ space, on T2-weighted images. ${ }^{3-5}$ It has been suggested that this accumulation of fluid could be a surrogate of an inflammatory process that may activate or sensitize nociceptive afferent neurons within the joint. ${ }^{4-6}$ Alternatively, it could result in increased intra-articular pressure, which may cause mechanical trauma, leading to hypoxia and other inflammation induced changes within the joint space, and eventually leads arthralgia. ${ }^{7}$ Several studies have been conducted on the association of joint effusion and TMJ pain associated with TMD. However, the results have been inconsistent. While the majority of studies have reported a statistically significant association, ${ }^{4-26}$ others have reported inconclusive findings. ${ }^{5-30}$ Due to this difference in reporting, a systematic review was conducted to determine if there is an association between joint effusion and self-reported TMJ-pain during examination, as well as between joint effusion and arthralgia as defined by the Research Diagnostic Criteria for Temporomandibular Disorders (RDC-TMD). ${ }^{31}$

\section{Materials and methods}

The clinical question: "Is there an association between MRI identified temporomandibular joint effusion and self-reported TMJpain?" was utilized to guide the review.

a. Inclusion Criteria: Observational studies evaluating a possible association between ipsilateral joint effusion, identified by MRI T2-weighted images, and TMJ pain were included. Manuscripts were limited to the English language only, published from inception till January 2017.

b. Exclusion Criteria: All investigations that included participants with rheumatologic diseases, or joint fractures were excluded. In addition, publications such as literature reviews and casereports were also excluded from the review. 
c. Literature Search: Four reviewers separately identified the pertinent literature by searching PubMed, MEDLINE and Cochrane databases under the inclusion criteria of all studies that have been conducted to determine an association between joint effusion and pain in the TMJ. The MeSH terms included TMJ, effusion, MRI, pain and arthralgia.

d. Procedure: Literature review was performed in accordance to PRISMA statement (Preferred Reporting Items for Systematic Reviews and Meta-Analyses).Four reviewers independently read the title and the abstract of all publications that matched the MeSH search terms. The reviewers met and developed a final list of publications to be read by consensus. Two reviewers read all the papers (SKN, RFGM); the other two reviewers (HC, YG) equally divided the number papers so that each paper was evaluated independently by three reviewers (Figure 1). Articles were reviewed based on the aim of the study, demographics, methodology, diagnostic criteria, MRI protocol, results, and conclusions.

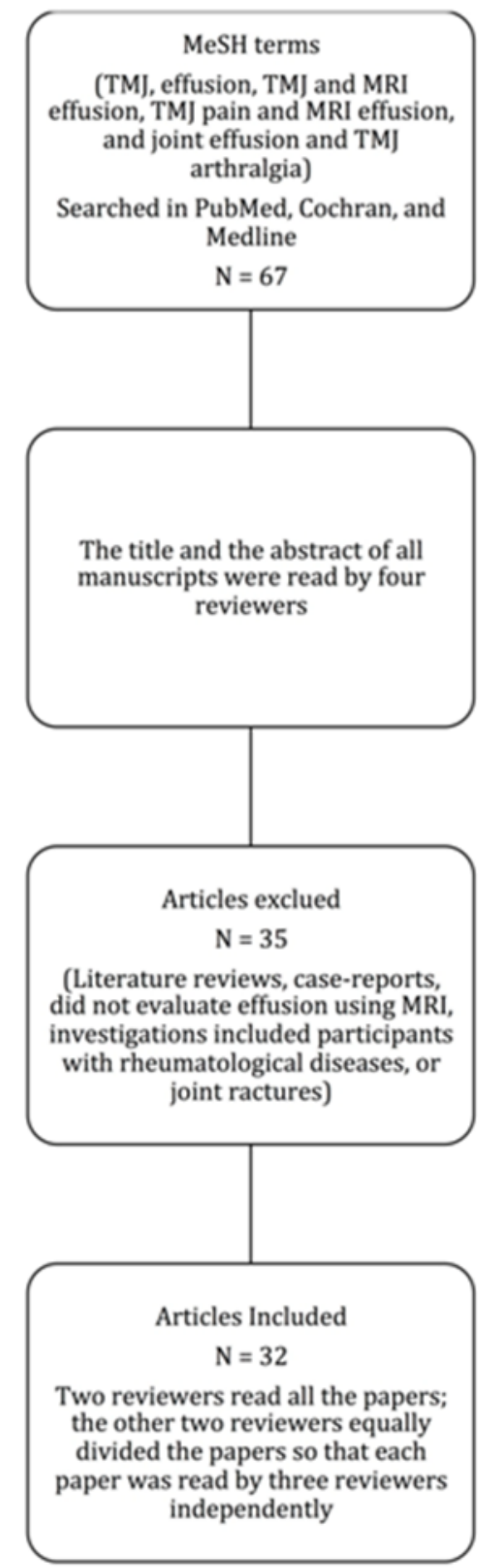

Figure I Flow chart summarizing the process of literature search. 


\section{Results}

Three databases (PubMed, Cochran, and Medline) were systematically searched for articles. A total of 67 articles were identified with the search strategy. Thirty-two met the inclusion criteria, and all were observational studies. Thirty-five publications were excluded because they did not evaluate the association of TMJ effusion with TMJ pain, did not use MRI to evaluate effusion, were case-reports, or literature review studies. The summary of 32 included articles is provided in Table 1. The majority of the included studies reported a statistically significant association between joint effusion and selfreported TMJ-pain. However, during the review of these studies, several methodological limitations were identified. For example: absence of control group; ${ }^{4-32}$ lack of information about examiner or radiologist reliability; ${ }^{10-26}$ failure to report details regarding the clinical examination; ${ }^{4-32}$ joint pain assessed by self-report (history) only without a clinical examination; ${ }^{15}$ or inappropriate statistical analysis. ${ }^{25}$ Among the studies that failed to find an association between TMJ effusion and self-reported TMJ-pain, several methodological limitations were also present. These include inappropriate control groups; ${ }^{5-30}$ lack of information about the evaluation of TMJ pain, or evaluation using a non-standardized examination; ${ }^{6-30}$ or lack of information regarding examiner and radiologist reliability. ${ }^{6-30}$

Table I Summary of the studies included in the systematic review on the association of TMJ effusion and TMJ pain

\begin{tabular}{lllll}
\hline $\begin{array}{l}\text { Author } \\
\text { and Year }\end{array}$ & Study aim & Demographics & $\begin{array}{l}\text { TMJ pain } \\
\text { assessment } \\
\text { methodology }\end{array}$ & $\begin{array}{l}\text { Imaging } \\
\text { assessment } \\
\text { methodology }\end{array}$ \\
\hline
\end{tabular}

$\begin{array}{ll} & \begin{array}{l}\text { Aim:To } \\ \text { determine the } \\ \text { clinical and } \\ \text { radiological } \\ \text { significance of JE } \\ \text { in patients with } \\ \text { TMD. }\end{array}\end{array}$

$59.4 \%$ 우

age: $3| \pm| I . \mid$ yrs.

$40.6 \%$ क

age: $29.8 \pm 9.7 \mathrm{yrs}$.

Oliveira et al. ${ }^{4}$

Aim:To evaluate
the association
between
condylar bone
changes and
presence of
TMJ effusion in
symptomatic
patients

Aim: To evaluate $68.9 \%$ 우 age: $40.4 \pm \mid 4.5$ yrs.

\section{Study group: \\ Participants underwent $\mathrm{DC} /$ TMD examination by calibrated examiners}

JE defined: presence of any high intensity signal in the superior or inferior joint spaces.

T2-weighted sagittal sequences ( $1.5 \mathrm{~T}$, $3 \mathrm{~mm}$ thick cuts)
Generalized estimating equation analysis T-test, Pearson Chi-Square,

ard

No association was determined between JE and TMJ arthralgia. Statistical association was determined between JR and dis position in the coronal and in the sagittal plan.

GEE suggested that disc displacement with reduction in the sagittal plan was statistically significant contributing factor for JE.
Pain was no categorized on basis of intensity.
Radiologist was calibrated.
T2-weighted sagittal sequences (I.5 $\mathrm{T}$, $3 \mathrm{~mm}$ thick cuts)

JE defined: any high signal intensity in the articular space.

Study group: Participants reported at least one sign or symptom of TMD during clinical examination
Two radiologist, diagnosis by consensus
Osteoarthritic changes were No control

associated with group JE.

Prevalence of JE Fisher's exact in symptomatic test patients was $10 \%$.
Clinical examination was not adequately described.

Reliability of clinical examiners and radiologists was not described. 
Table Continued...

\begin{tabular}{|c|c|c|c|c|c|c|c|}
\hline $\begin{array}{l}\text { Author } \\
\text { and Year }\end{array}$ & Study aim & Demographics & $\begin{array}{l}\text { TMJ pain } \\
\text { assessment } \\
\text { methodology }\end{array}$ & $\begin{array}{l}\text { Imaging } \\
\text { assessment } \\
\text { methodology }\end{array}$ & Statistics & Conclusion & Limitations \\
\hline \multirow[t]{3}{*}{$\begin{array}{l}\text { Lamot } U \text { et } \\
\text { al. } .^{14}\end{array}$} & Aim: & $\mathrm{N}=104 ; 288 \mathrm{TMJs}$ & \multirow{3}{*}{$\begin{array}{l}\text { Study group: } \\
\text { Participants had } \\
\text { unilateral or bilateral } \\
\text { presence of TMJ } \\
\text { pain, noises/clicking, } \\
\text { limited movement } \\
\text { and headache. Study } \\
\text { group was divided } \\
\text { into } 3 \text { sub-groups } \\
\text { based on type of ID. }\end{array}$} & $\begin{array}{l}\text { T2-weighted } \\
\text { sagittal } \\
\text { sequences ( } 1.5 \mathrm{~T} \text {, } \\
20 \text { slices) }\end{array}$ & Prevalence & $\begin{array}{l}\text { Morphological } \\
\text { changes } \\
\text { associated with } \\
\text { symptoms of } \\
\text { TMJ dysfunction. } \\
\text { Prevalence of JE } \\
\text { in symptomatic } \\
\text { joints was I4.6 } \\
\%\end{array}$ & $\begin{array}{l}\text { Clinical } \\
\text { examination } \\
\text { was not } \\
\text { adequately } \\
\text { described. }\end{array}$ \\
\hline & $\begin{array}{l}\text { a. To determine } \\
\text { correlation } \\
\text { between MRI and } \\
\text { clinical findings } \\
\text { associated with } \\
\text { TMD. }\end{array}$ & $75.7 \%$ \% ; $24.3 \%$ ठ & & $\begin{array}{l}\text { JE defined: } \\
\text { any high signal } \\
\text { intensity in } \\
\text { anterior or } \\
\text { posterior recess, } \\
\text { or large amount }\end{array}$ & $\begin{array}{l}\text { Fisher's exact } \\
\text { test }\end{array}$ & $\begin{array}{l}\text { Younger age } \\
\text { group was } \\
\text { associated with } \\
\text { JE }\end{array}$ & $\begin{array}{l}\text { Reliability } \\
\text { of clinical } \\
\text { examiners and } \\
\text { radiologists } \\
\text { was not } \\
\text { described }\end{array}$ \\
\hline & $\begin{array}{l}\text { b. To assess the } \\
\text { impact of gender } \\
\text { and age, on this } \\
\text { correlation. }\end{array}$ & Total sample & & $\begin{array}{l}\text { in superior or } \\
\text { inferior joint } \\
\text { space. }\end{array}$ & T-Test & & $\begin{array}{l}\text { Radiologists } \\
\text { were not blind } \\
\text { to the clinical } \\
\text { diagnosis }\end{array}$ \\
\hline
\end{tabular}

Santos et al. $^{20}$ age: 39.4 yrs. $(12-$ 81 yrs.).

$N=7 \mid$; I42 TMJs

Aim:To assess

the association

between changes

of the articular

eminence and

the condyle,

articular disc

changes, and

the presence of

TMJ effusion in

symptomatic

patients.

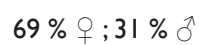

Study group:

Participants had resence of TM roup was divided into 3 sub-groups anterior or posterior recess, inferior joint

T2-weighted sagittal sequences (I.5 T, $3 \mathrm{~mm}$ thick cuts)

JE defined: any hyper-signal in the articular space.

Two radiologist, diagnosis by consensus
Association was present between: I) disc form and its position; 2) condylar form and an anterior portion of disc; and 3) articular eminence form and disc form.

Prevalence of JE

Clinical

Pearson's Chiin symptomatic Square test joints was 23.9 $\%$.

No control group examination was not adequately described.

Reliability of clinical examiners and radiologists was not described 
Table Continued...

Author and Year
Demographics
TMJ pain

assessment

methodology
Imaging

assessment

methodology
Statistics

Conclusion

Limitations

\begin{tabular}{|c|c|c|c|}
\hline Park et al. ${ }^{18}$ & & $\mathrm{~N}=100 ; 200 \mathrm{TMJs}$ & $\begin{array}{l}\text { Study group: } \\
\text { Participants } \\
\text { underwent RDC/ } \\
\text { TMD examination. }\end{array}$ \\
\hline & $\begin{array}{l}\text { Aim:To } \\
\text { investigate the } \\
\text { value of MRI in } \\
\text { the diagnostic } \\
\text { process based on } \\
\text { the RDC/TMD. }\end{array}$ & $68.0 \% q$ with & \\
\hline & & age: $25.3 \pm 13.12$ yrs. & $\begin{array}{l}\text { Study group was } \\
\text { divided into } 3 \\
\text { distinct Group II } \\
\text { diagnoses based on } \\
\text { RDC/TMD. }\end{array}$ \\
\hline
\end{tabular}

$32 \%$ with mean age: $31.9 \pm 12.3 \mathrm{yrs}$

Bas et al. ${ }^{9}$
$\mathrm{N}=91 ;$ | $82 \mathrm{TM} \mathrm{J}$

Study group

Aim:To evaluate the relationship between the grades of MRI depicted JE, increased capsular width (CW) measured in Ultrasonographic Imaging (USI), and joint pain.

\section{$81.3 \%$}

Participants underwent

a. RDC/TMD
examination.

$18.7 \%$

Total sample
JE defined: any high intensity signal in articular surface or compartment.

Two radiologist, diagnosis by consensus

Radiologist underwent calibration.

T2-weighted sagittal sequences (0.5 T, $3 \mathrm{~mm}$ thick cuts)

JE defined: area of homogenous and high signal intensity.

Friedman Test

Statistically significant association was found between VAS score and intensity of MRI- JE.

JE was

characterized

into moderate Wilcoxon Test JE absent: 2.55 and severe effusion.

b.VAS to measure $\quad \mathrm{CW}$ was pain intensity. calculated to be
Moderate JE:

2.92
Cohen's Kappa showed no

agreement

to moderate

agreement

and Group II

diagnosis based

No

information

if radiologists were blind to the clinical diagnosis

$43.4 \%$ of joints with pain had JE while $28.7 \%$ of non-painful joints had JE

Reliability

of clinical examiners and radiologists

was not reported.

Receiver Operating Characteristic CW $1.65 \mathrm{~mm}$

No control group

\section{Reliability of clinical examiners and radiologists was not described}

Severe JE: 4.80 
Table Continued...

\begin{tabular}{|c|c|c|c|c|c|c|c|}
\hline $\begin{array}{l}\text { Author } \\
\text { and Year }\end{array}$ & Study aim & Demographics & $\begin{array}{l}\text { TMJ pain } \\
\text { assessment } \\
\text { methodology }\end{array}$ & $\begin{array}{l}\text { Imaging } \\
\text { assessment } \\
\text { methodology }\end{array}$ & Statistics & Conclusion & Limitations \\
\hline \multirow[t]{2}{*}{$\begin{array}{l}\text { Kaneyama et } \\
\text { al. }^{13}\end{array}$} & \multirow[t]{2}{*}{$\begin{array}{l}\text { Aim: To } \\
\text { determine the } \\
\text { association } \\
\text { between JE } \\
\text { and various } \\
\text { soluble cytokine } \\
\text { receptors in the } \\
\text { synovial fluid. }\end{array}$} & $\mathrm{N}=55 ; 55 \mathrm{TMJs}$ & $\begin{array}{l}\text { Study group: } \\
\text { Participants had } \\
\text { diagnosis of TMD } \\
\text { based on }\end{array}$ & $\begin{array}{l}\text { T2-weighted } \\
\text { sagittal } \\
\text { sequences (I.5 T, } \\
\text { 3mm thick cuts) } \\
\text { JE defined: area } \\
\text { of high intensity } \\
\text { signal in superior } \\
\text { compartment. } \\
\text { JE was } \\
\text { characterized } \\
\text { into grade I, II, } \\
\text { and III based on } \\
\text { the quantity of } \\
\text { signal. }\end{array}$ & $\begin{array}{l}\text { Mann-Whitney } \\
\text { U test }\end{array}$ & $\begin{array}{l}\text { The mean } \\
\text { concentration } \\
\text { of cytokine } \\
\text { receptors in the } \\
\text { synovial fluid } \\
\text { were significantly } \\
\text { higher in joints } \\
\text { with JE than in } \\
\text { joints without JE. }\end{array}$ & $\begin{array}{l}\text { Clinical } \\
\text { examination } \\
\text { was not } \\
\text { adequately } \\
\text { described. }\end{array}$ \\
\hline & & & $\begin{array}{l}\text { b.VAS used to } \\
\text { measure ipsilateral } \\
\text { joint pain intensity. }\end{array}$ & & & $\begin{array}{l}\text { No statistically } \\
\text { significant } \\
\text { difference } \\
\text { was observed } \\
\text { between severity } \\
\text { of JE and VAS. }\end{array}$ & $\begin{array}{l}\text { Reliability } \\
\text { of clinical } \\
\text { examiners and } \\
\text { radiologists } \\
\text { was not } \\
\text { described. } \\
\text { High signal in } \\
\text { the inferior } \\
\text { TM joint } \\
\text { compartment } \\
\text { was not } \\
\text { considered } \\
\text { as indicative } \\
\text { of JE. }\end{array}$ \\
\hline \multirow[t]{3}{*}{$\begin{array}{l}\text { Nakaoka et } \\
\text { al. }^{32}\end{array}$} & $\begin{array}{l}\text { Aim:To } \\
\text { investigate the } \\
\text { changes of JE } \\
\text { on the MRI } \\
\text { and pathology } \\
\text { observed in } \\
\text { arthroscopy after } \\
\text { VGIR. }\end{array}$ & $\mathrm{N}=56 ; 56 \mathrm{TMJs}$ & $\begin{array}{l}\text { Study group: } \\
\text { Participants } \\
\text { underwent clinical } \\
\text { examination. }\end{array}$ & $\begin{array}{l}\text { T2-weighted } \\
\text { sagittal } \\
\text { sequences }(0.3 \mathrm{~T})\end{array}$ & $\begin{array}{l}\text { Wilcoxon } \\
\text { signed-rank } \\
\text { sum test }\end{array}$ & $\begin{array}{l}\text { JE was reported } \\
\text { in } 65 \% \text { of joints. }\end{array}$ & $\begin{array}{l}\text { Gender } \\
\text { demographics } \\
\text { not provided. }\end{array}$ \\
\hline & $\begin{array}{l}\text { a.To correlate } \\
\text { these findings } \\
\text { with clinical } \\
\text { variables. }\end{array}$ & $\begin{array}{l}40 \text { participants } \\
\text { completed the study }\end{array}$ & $\begin{array}{l}\text { Study group had a } \\
\text { clinical diagnosis of } \\
\text { unilateral chronic } \\
\text { closed lock. }\end{array}$ & $\begin{array}{l}\text { JE was } \\
\text { characterized } \\
\text { into grade I, II, } \\
\text { and III. }\end{array}$ & $\begin{array}{l}\text { Mann-Whitney } \\
\text { U test }\end{array}$ & $\begin{array}{l}\text { No statistical } \\
\text { correlation } \\
\text { between JE and } \\
\text { synovial lining } \\
\text { score. }\end{array}$ & $\begin{array}{l}\text { Clinical } \\
\text { examination } \\
\text { was not } \\
\text { adequately } \\
\text { described. }\end{array}$ \\
\hline & & age 43 yrs. & & $\begin{array}{l}\text { Three radiologist, } \\
\text { diagnosis by } \\
\text { consensus }\end{array}$ & & & $\begin{array}{l}\text { Reliability } \\
\text { of clinical } \\
\text { examiners and } \\
\text { radiologists } \\
\text { was not } \\
\text { described. }\end{array}$ \\
\hline
\end{tabular}


Table Continued.

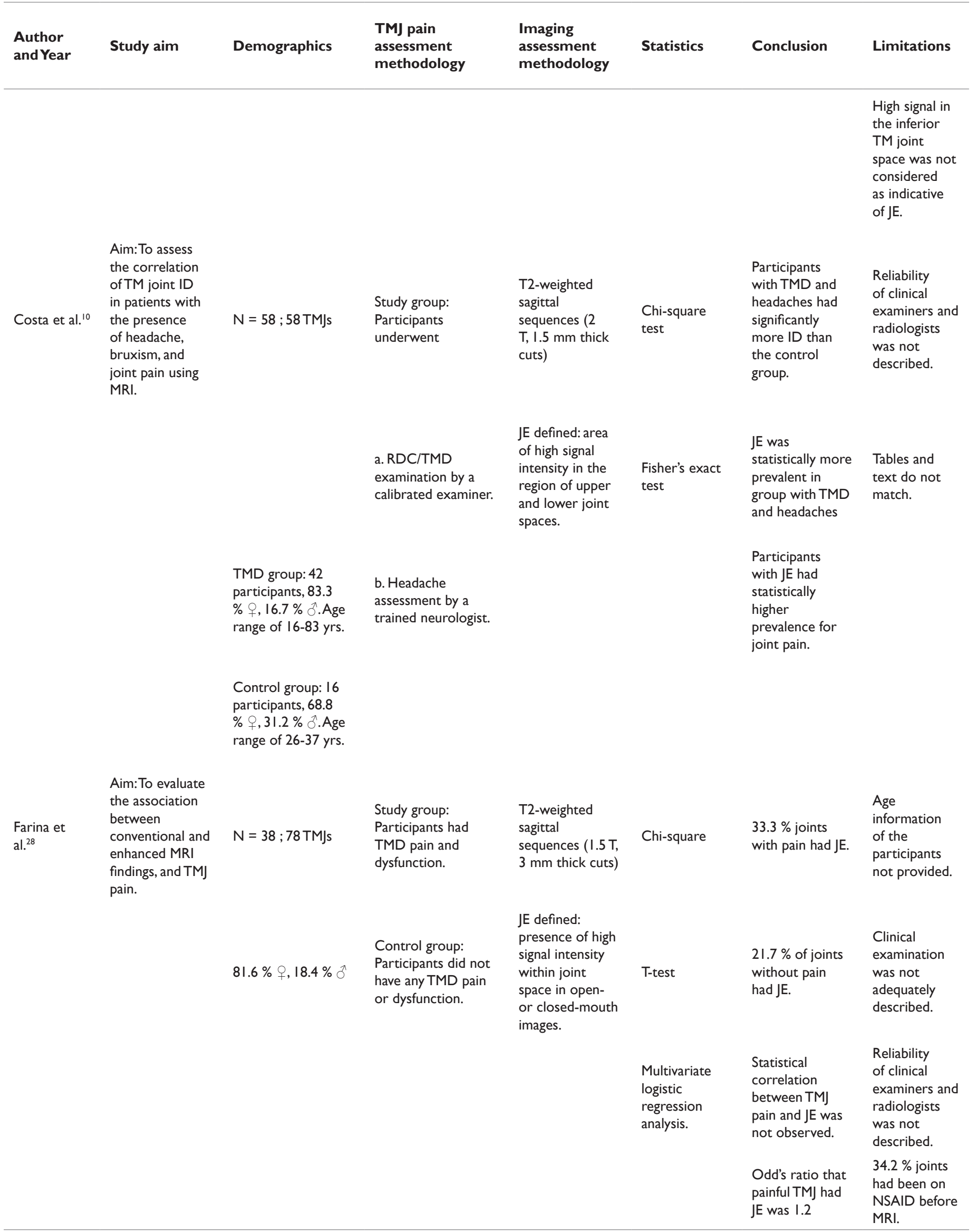


Table Continued...

\begin{tabular}{|c|c|c|c|c|c|c|c|}
\hline $\begin{array}{l}\text { Author } \\
\text { and Year }\end{array}$ & Study aim & Demographics & $\begin{array}{l}\text { TMJ pain } \\
\text { assessment } \\
\text { methodology }\end{array}$ & $\begin{array}{l}\text { Imaging } \\
\text { assessment } \\
\text { methodology }\end{array}$ & Statistics & Conclusion & Limitations \\
\hline \multirow[t]{4}{*}{$\begin{array}{l}\text { Limchaichana } \\
\text { et al. } .^{5}\end{array}$} & $\begin{array}{l}\text { Aim:To evaluate } \\
\text { the association } \\
\text { between MRI } \\
\text { findings and } \\
\text { clinical symptoms } \\
\text { of TMD. }\end{array}$ & $\mathrm{N}=60 ; \mid 20 \mathrm{TM} \mathrm{s}$ & $\begin{array}{l}\text { Study group: } \\
\text { Participants } \\
\text { underwent RDC/ } \\
\text { TMD examination } \\
\text { by a calibrated } \\
\text { examiner. }\end{array}$ & $\begin{array}{l}\text { T2-weighted } \\
\text { sagittal } \\
\text { sequences }(1.5 \mathrm{~T})\end{array}$ & T-test & $\begin{array}{l}\text { I8 \% of joints } \\
\text { with myofascial } \\
\text { pain only, had JE. }\end{array}$ & $\begin{array}{l}\text { Overlapping } \\
\text { of diagnosis } \\
\text { - both } \\
\text { groups had } \\
\text { masticatory } \\
\text { muscle pain. }\end{array}$ \\
\hline & & $78.3 \%$ \%, $21.7 \%$ ○ & Divided into & $\begin{array}{l}\text { JE defined: area } \\
\text { of more than } \\
\text { a line or dots } \\
\text { of high signal } \\
\text { intensity in } \\
\text { the region of } \\
\text { superior or } \\
\text { inferior joint } \\
\text { space. }\end{array}$ & $\begin{array}{l}\text { Fisher's exact } \\
\text { test }\end{array}$ & $\begin{array}{l}33 \% \text { of joints } \\
\text { with myofascial } \\
\text { pain and } \\
\text { arthralgia/OA } \\
\text { had JE. }\end{array}$ & \\
\hline & & $\begin{array}{l}\text { No difference } \\
\text { between the groups } \\
\text { in age and gender } \\
\text { was found. }\end{array}$ & $\begin{array}{l}\text { a. Myofascial pain } \\
\text { only. }\end{array}$ & $\begin{array}{l}\text { Radiologist had } \\
\text { inter-observer } \\
\text { agreement of } \\
0.69 \text { for JE }\end{array}$ & $\begin{array}{l}\text { Chi-square } \\
\text { test }\end{array}$ & $\begin{array}{l}\text { No statistical } \\
\text { difference } \\
\text { between two } \\
\text { groups in } \\
\text { prevalence of JE. }\end{array}$ & \\
\hline & & & $\begin{array}{l}\text { b. Myofascial pain } \\
\text { with Arthralgia/OA. }\end{array}$ & & Kappa statistic & & \\
\hline \multirow[t]{6}{*}{ Guler et al. ${ }^{29}$} & $\begin{array}{l}\text { Aim:To } \\
\text { determine the } \\
\text { association } \\
\text { between } \\
\text { JE, protein } \\
\text { concentration in } \\
\text { synovial fluid, and } \\
\text { TMJ pain. }\end{array}$ & $\mathrm{N}=31 ; 31 \mathrm{TMJs}$ & $\begin{array}{l}\text { Study group: } \\
\text { Participants } \\
\text { underwent }\end{array}$ & $\begin{array}{l}\text { T2-weighted } \\
\text { sagittal } \\
\text { sequences (I.5 } \\
\text { T, } 3 \text { mm thick } \\
\text { slices) }\end{array}$ & Chi-square & $\begin{array}{l}7.7 \% \text { joints in } \\
\text { control group } \\
\text { had JE. }\end{array}$ & $\begin{array}{l}\text { Reliability } \\
\text { of clinical } \\
\text { examiners and } \\
\text { radiologists } \\
\text { was not } \\
\text { described. }\end{array}$ \\
\hline & & $\begin{array}{l}\text { Study group: } 81.3 \% \\
+, 18.7 \% \delta .\end{array}$ & $\begin{array}{l}\text { a. RDC/TMD } \\
\text { examination. }\end{array}$ & $\begin{array}{l}\text { JE defined: high- } \\
\text { signal area in the } \\
\text { upper and lower } \\
\text { joint spaces. }\end{array}$ & $\begin{array}{l}\text { Spearman's } \\
\text { rank } \\
\text { correlation }\end{array}$ & $\begin{array}{l}75 \% \text { joints in } \\
\text { study group } \\
\text { had JE. }\end{array}$ & \\
\hline & & $\begin{array}{l}\text { age: } 31 \text { yrs. (I7-57 } \\
\text { yrs.) }\end{array}$ & $\begin{array}{l}\text { b.VAS to measure } \\
\text { pain intensity. }\end{array}$ & $\begin{array}{l}\text { JE was } \\
\text { characterized } \\
\text { into } 4 \text { levels } \\
\text { depending on } \\
\text { quantity of joint } \\
\text { fluid. }\end{array}$ & & $\begin{array}{l}\text { No significant } \\
\text { association } \\
\text { was observed } \\
\text { between level of } \\
\text { pain and JE. }\end{array}$ & \\
\hline & & $\begin{array}{l}\text { Control group: } 66.7 \\
\%+, 33.3 \%\end{array}$ & $\begin{array}{l}\text { Participants were } \\
\text { divided into: }\end{array}$ & & & & \\
\hline & & $\begin{array}{l}\text { age: } 28 \text { yrs. (17-42 } \\
\text { yrs.) }\end{array}$ & $\begin{array}{l}\text { a. DDwoR, with } \\
\text { arthralgia and OA. }\end{array}$ & & & & \\
\hline & & & $\begin{array}{l}\text { b. DDwoR without } \\
\text { any pain-related } \\
\text { TMD diagnosis. }\end{array}$ & & & & \\
\hline
\end{tabular}


Table Continued.

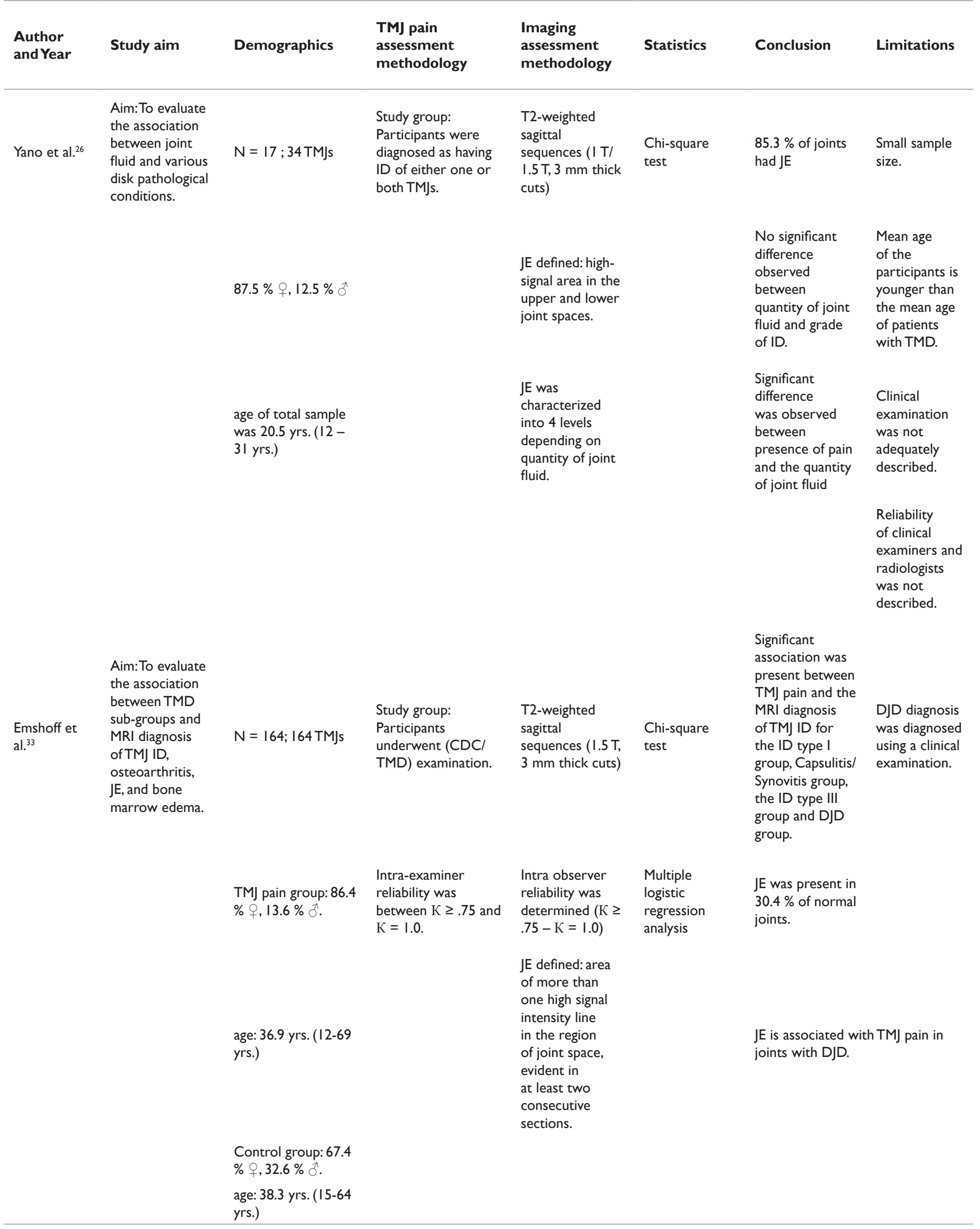


Table Continued...

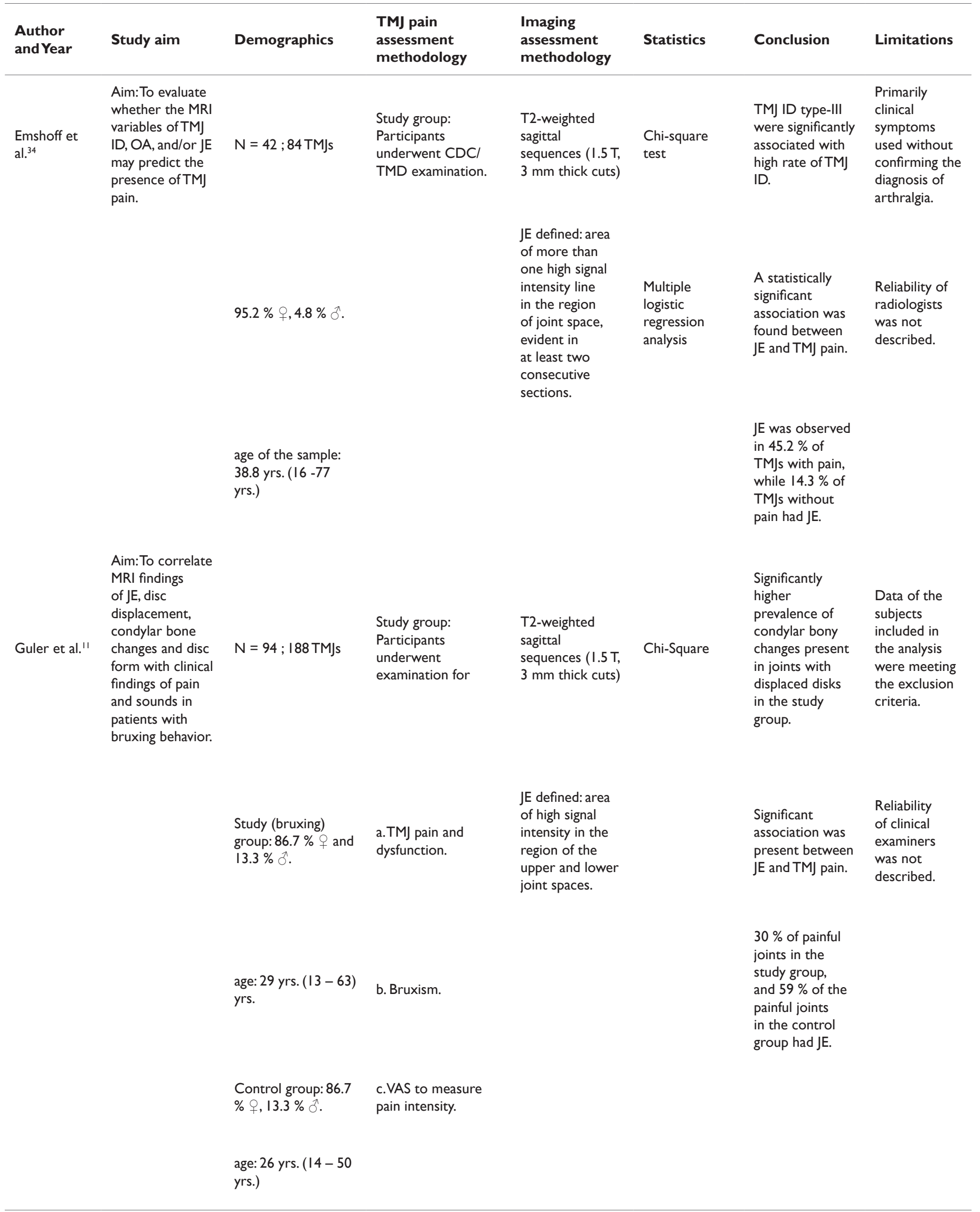


Table Continued...

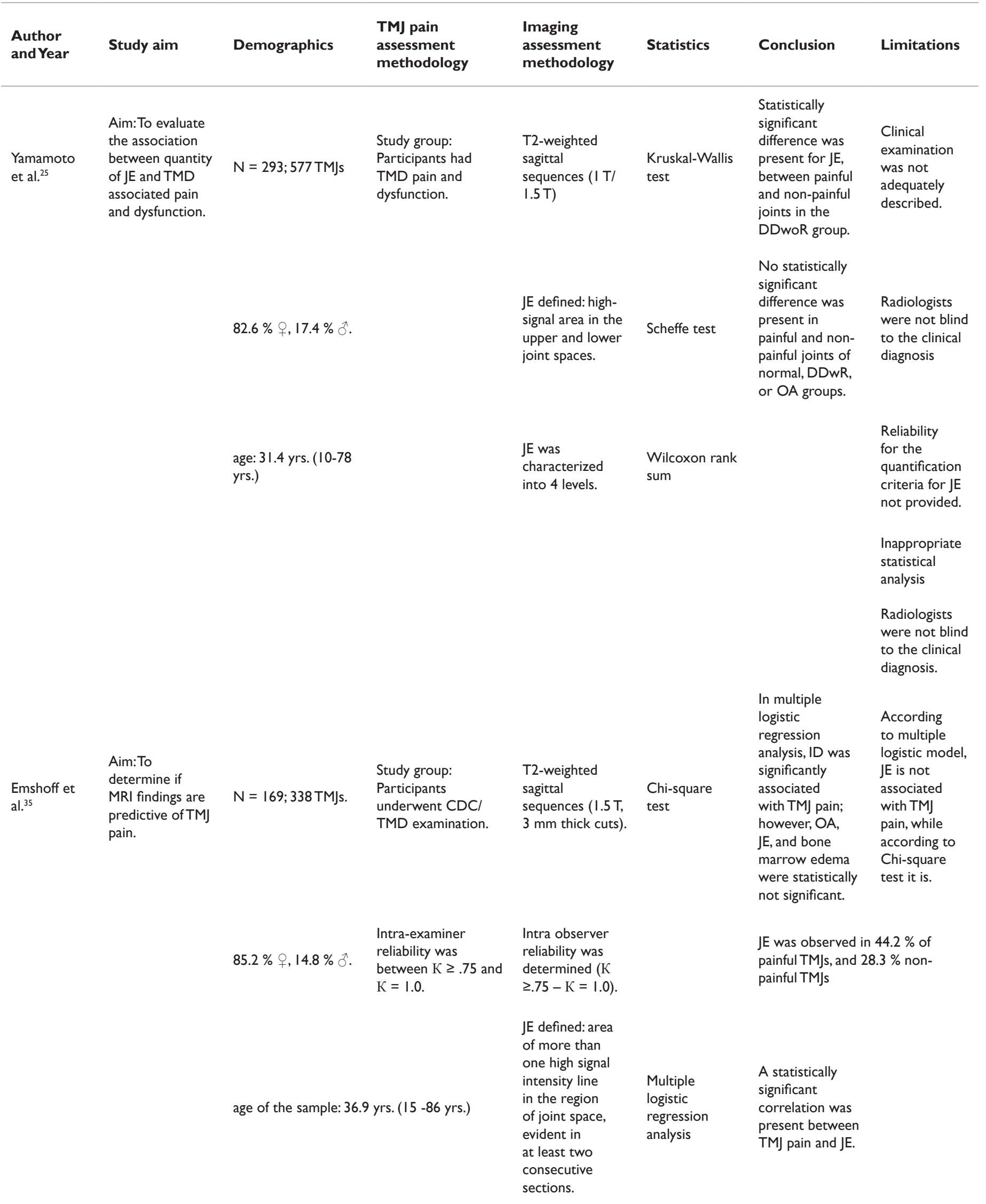


Table Continued..

\begin{tabular}{|c|c|c|c|c|c|c|c|}
\hline $\begin{array}{l}\text { Author } \\
\text { and Year }\end{array}$ & Study aim & Demographics & $\begin{array}{l}\text { TMJ pain } \\
\text { assessment } \\
\text { methodology }\end{array}$ & $\begin{array}{l}\text { Imaging } \\
\text { assessment } \\
\text { methodology }\end{array}$ & Statistics & Conclusion & Limitations \\
\hline \multirow[t]{3}{*}{$\begin{array}{l}\text { Manfredini } \\
\text { et al. }{ }^{16}\end{array}$} & \multirow[t]{3}{*}{$\begin{array}{l}\text { Aim:To evaluate } \\
\text { the predictive } \\
\text { value of clinical } \\
\text { symptoms for } \\
\text { MRI findings } \\
\text { of JE. }\end{array}$} & \multirow[t]{3}{*}{$N=6 I$} & $\begin{array}{l}\text { Study group: } \\
\text { Participants } \\
\text { underwent }\end{array}$ & $\begin{array}{l}\text { T2-weighted } \\
\text { sagittal } \\
\text { sequences ( } 0.5 \mathrm{~T} \text {, } \\
3 \text { mm thick cuts) }\end{array}$ & $\begin{array}{l}\text { Multiple } \\
\text { logistic analysis }\end{array}$ & $\begin{array}{l}\text { Except for pain } \\
\text { during joint } \\
\text { play and pain } \\
\text { in the TM joint } \\
\text { during dynamic } \\
\text { tests, rest of the } \\
\text { clinical variables } \\
\text { were included } \\
\text { in the logistic } \\
\text { regression } \\
\text { analysis. }\end{array}$ & $\begin{array}{l}\text { Gender } \\
\text { demographics } \\
\text { were not } \\
\text { provided. }\end{array}$ \\
\hline & & & $\begin{array}{l}\text { a. RDC/TMD } \\
\text { examination. }\end{array}$ & $\begin{array}{l}\text { JE defined: area } \\
\text { of high signal } \\
\text { intensity inside } \\
\text { the joint space. }\end{array}$ & $\begin{array}{l}\text { Goodness-of- } \\
\text { fit test }\end{array}$ & $\begin{array}{l}\text { Among single } \\
\text { parameters, } \\
\text { presence of pain } \\
\text { in the TMJ with } \\
\text { lateral palpation } \\
\text { provided good } \\
\text { diagnostic } \\
\text { accuracy of } \\
76.2 \% \text {. }\end{array}$ & $\begin{array}{l}\text { No control } \\
\text { group. }\end{array}$ \\
\hline & & & $\begin{array}{l}\text { b.Additional clinical } \\
\text { examination (pain } \\
\text { in TMJ during } \\
\text { posterior palpation, } \\
\text { dynamic tests). }\end{array}$ & & $\begin{array}{l}\text { Model Chi- } \\
\text { square test }\end{array}$ & & $\begin{array}{l}\text { Reliability of } \\
\text { radiologists } \\
\text { was not } \\
\text { described. }\end{array}$ \\
\hline \multirow[t]{4}{*}{$\begin{array}{l}\text { Emshoff et } \\
\text { al. }{ }^{36}\end{array}$} & $\begin{array}{l}\text { Aim: To evaluate } \\
\text { the association } \\
\text { between MRI } \\
\text { findings of OA, } \\
\text { and/or JE, and } \\
\text { TMJ pain. }\end{array}$ & $\mathrm{N}=\mathrm{I} I 2 ; 224 \mathrm{TM} \mathrm{Js}$ & $\begin{array}{l}\text { Study group: } \\
\text { Participants } \\
\text { underwent CDC/ } \\
\text { TMD examination. }\end{array}$ & $\begin{array}{l}\text { T2-weighted } \\
\text { sagittal } \\
\text { sequences ( } 1.5 \mathrm{~T} \text {, } \\
3 \text { mm thick cuts) }\end{array}$ & Chi-square & $\begin{array}{l}88.4 \% \text { of joints } \\
\text { with TMJ-Pain, } \\
\text { and } 66.1 \% \text { of } \\
\text { joints without } \\
\text { TMJ-Pain had } \\
\text { OA. }\end{array}$ & $\begin{array}{l}\text { Reliability } \\
\text { of clinical } \\
\text { examiners and } \\
\text { radiologists } \\
\text { was not } \\
\text { described. }\end{array}$ \\
\hline & & $86.6 \%+, 13.4 \% \pi$ & & \multirow[t]{3}{*}{$\begin{array}{l}\text { JE defined: area } \\
\text { of more than } \\
\text { one high signal } \\
\text { intensity line } \\
\text { in the region } \\
\text { of joint space, } \\
\text { evident in } \\
\text { at least two } \\
\text { consecutive } \\
\text { sections. }\end{array}$} & $\begin{array}{l}\text { Kappa statistic } \\
\text { test }\end{array}$ & $\begin{array}{l}48.2 \% \text { of joints } \\
\text { with TMJ-Pain, } \\
\text { and } 19.6 \% \text { of } \\
\text { joints without } \\
\text { TMJ-Pain had JE. }\end{array}$ & \\
\hline & & \multirow[t]{2}{*}{$\begin{array}{l}\text { age: } 38.2 \text { yrs. (15-78 } \\
\text { yrs.) }\end{array}$} & & & & $\begin{array}{l}\text { TMJ-Pain was } \\
\text { statistically } \\
\text { associated with } \\
\text { OA, and JE. }\end{array}$ & \\
\hline & & & & & & $\begin{array}{l}\text { Kappa value for } \\
\text { association of } \\
\text { TMJ-Pain and JE } \\
\text { was } 0.29 \text { (poor). }\end{array}$ & \\
\hline
\end{tabular}


Table Continued.

\begin{tabular}{|c|c|c|c|c|c|c|c|}
\hline $\begin{array}{l}\text { Author } \\
\text { and Year }\end{array}$ & Study aim & Demographics & $\begin{array}{l}\text { TMJ pain } \\
\text { assessment } \\
\text { methodology }\end{array}$ & $\begin{array}{l}\text { Imaging } \\
\text { assessment } \\
\text { methodology }\end{array}$ & Statistics & Conclusion & Limitations \\
\hline \multirow[t]{5}{*}{$\begin{array}{l}\text { Segami et } \\
\text { al. } .^{30}\end{array}$} & $\begin{array}{l}\text { Aim: To evaluate } \\
\text { the association } \\
\text { between JE and } \\
\text { synovial fluid } \\
\text { constituents in } \\
\text { patients with ID } \\
\text { and OA. }\end{array}$ & $\mathrm{N}=108 ; 108 \mathrm{TM} \mathrm{s}$ & $\begin{array}{l}\text { Study group: } \\
\text { Participant } \\
\text { underwent }\end{array}$ & $\begin{array}{l}\text { T2-wighted } \\
\text { sagittal } \\
\text { sequences ( } 1.5 \mathrm{~T} \text {, } \\
3 \mathrm{~mm} \text { thick } \\
\text { slices). }\end{array}$ & $\begin{array}{l}\text { Mann-Whitney } \\
\text { U test }\end{array}$ & $\begin{array}{l}\text { No statistically } \\
\text { significant } \\
\text { difference was } \\
\text { observed in pain } \\
\text { intensity among } \\
\text { study groups. }\end{array}$ & $\begin{array}{l}\text { Control group } \\
\text { only had } \delta \text {. }\end{array}$ \\
\hline & & $\begin{array}{l}\text { Study group: } 90 \% \\
\text {, } 10 \% \pi .\end{array}$ & a. MRI & $\begin{array}{l}\text { JE defined: high- } \\
\text { signal area in the } \\
\text { upper and lower } \\
\text { joint spaces. } \\
\text { It was further } \\
\text { characterized } \\
\text { into } 4 \text { levels } \\
\text { depending on } \\
\text { quantity of joint } \\
\text { fluid. }\end{array}$ & & $\begin{array}{l}\text { Joints with JE } \\
\text { had significantly } \\
\text { higher total } \\
\text { protein } \\
\text { concentration, } \\
\text { and Interleukin } \\
-6 \text { levels than } \\
\text { joints without JE. }\end{array}$ & $\begin{array}{l}\text { Clinical } \\
\text { examination } \\
\text { was not } \\
\text { adequately } \\
\text { described. }\end{array}$ \\
\hline & & $\begin{array}{l}\text { age: } 34.9 \text { yrs. (13-73 } \\
\text { yrs.) }\end{array}$ & $\begin{array}{l}\text { b.VAS to measure } \\
\text { pain intensity. }\end{array}$ & $\begin{array}{l}\text { Grade } 0 \text { and I } \\
\text { were excluded. }\end{array}$ & & & $\begin{array}{l}30 \% \\
\text { participants in } \\
\text { study group } \\
\text { were on } \\
\text { NSAID. }\end{array}$ \\
\hline & & $\begin{array}{l}\text { Control group: } \\
\text { All } \hat{\delta} \text {. }\end{array}$ & $\begin{array}{l}\text { Study group had } \\
\text { ID or OA, with or } \\
\text { without JE. }\end{array}$ & & & & $\begin{array}{l}\text { Reliability } \\
\text { of clinical } \\
\text { examiners and } \\
\text { radiologists } \\
\text { was not } \\
\text { described. }\end{array}$ \\
\hline & & age 30 yrs. & $\begin{array}{l}\text { Control group were } \\
\text { symptom free. }\end{array}$ & & & & \\
\hline \multirow[t]{5}{*}{$\begin{array}{l}\text { Tanaka et } \\
\text { al. }^{23}\end{array}$} & Aim: & $\mathrm{N}=200 ; 400 \mathrm{TMJ}$ & $\begin{array}{l}\text { Study group: } \\
\text { Participants } \\
\text { underwent RDC/ } \\
\text { TMD examination. }\end{array}$ & $\begin{array}{l}\text { FS T2-weighted } \\
\text { sagittal } \\
\text { sequences (I.5 T, } \\
3 \text { mm thick cuts). }\end{array}$ & $\begin{array}{l}\text { Chi-square } \\
\text { test }\end{array}$ & $\begin{array}{l}\text { Detection rate } \\
\text { of JE by FS was } \\
\text { significantly } \\
\text { greater than by } \\
\text { T2-weighted } \\
\text { MRI. }\end{array}$ & $\begin{array}{l}\text { Clinical } \\
\text { examination } \\
\text { was not } \\
\text { adequately } \\
\text { described. }\end{array}$ \\
\hline & $\begin{array}{l}\text { a. To compare } \\
\text { JE evaluation } \\
\text { between } \\
\text { T2-weighted } \\
\text { MRI and Fat } \\
\text { saturation (FS) } \\
\text { MRI. }\end{array}$ & $76.5 \%$ \%, $23.5 \% \delta$ & & $\begin{array}{l}\text { T2-weighted } \\
\text { sagittal } \\
\text { sequences ( } 1.5 \mathrm{~T} \text {, } \\
3 \text { mm thick cuts). }\end{array}$ & $\begin{array}{l}\text { Kappa statistic } \\
\text { test }\end{array}$ & $\begin{array}{l}\text { Statistically } \\
\text { significant } \\
\text { association } \\
\text { between JE and } \\
\text { TMJ pain. }\end{array}$ & $\begin{array}{l}\text { Reliability of } \\
\text { radiologists } \\
\text { was not } \\
\text { described. }\end{array}$ \\
\hline & $\begin{array}{l}\text { b. To determine } \\
\text { association } \\
\text { between JE and } \\
\text { MRI findings. }\end{array}$ & $\begin{array}{l}\text { age of case group: } \\
29.3 \text { yrs. (I } 7-52 \text { yrs.) }\end{array}$ & & $\begin{array}{l}\text { JE defined: area } \\
\text { of high signal } \\
\text { intensity greater } \\
\text { than a moderate } \\
\text { amount of fluid. }\end{array}$ & & $\begin{array}{l}\text { Kappa test } \\
\text { indicates good } \\
\text { agreement } \\
\text { between pain } \\
\text { and JE (K = } \\
0.66) \text {. }\end{array}$ & \\
\hline & & $\begin{array}{l}20 \text { participants were } \\
\text { in control group. }\end{array}$ & & & & $\begin{array}{l}\text { FS indicates } \\
70 \% \text { of painful } \\
\text { joints and } 3 \% \text { of } \\
\text { pain-free joints } \\
\text { having JE. }\end{array}$ & \\
\hline & & $\begin{array}{l}65 \% \text { were }+, 35 \\
\% \text {. } \\
\text { age of control } \\
\text { group: } 23.3 \text { yrs. (18- } \\
35 \text { yrs.) }\end{array}$ & & & & & \\
\hline
\end{tabular}


Table Continued...

\begin{tabular}{|c|c|c|c|c|c|c|c|}
\hline $\begin{array}{l}\text { Author } \\
\text { and Year }\end{array}$ & Study aim & Demographics & $\begin{array}{l}\text { TMJ pain } \\
\text { assessment } \\
\text { methodology }\end{array}$ & $\begin{array}{l}\text { Imaging } \\
\text { assessment } \\
\text { methodology }\end{array}$ & Statistics & Conclusion & Limitations \\
\hline \multirow[t]{2}{*}{ Haley et al. ${ }^{12}$} & $\begin{array}{l}\text { Aim:To } \\
\text { investigate the } \\
\text { association } \\
\text { between TMJ- } \\
\text { Pain and clinical } \\
\text { and MRI findings. }\end{array}$ & N = 85; I 70 TMJs. & $\begin{array}{l}\text { Study group: } \\
\text { Participants } \\
\text { underwent } \\
\text { standardized clinical } \\
\text { examination. }\end{array}$ & $\begin{array}{l}\text { T2-weighted } \\
\text { sagittal } \\
\text { sequences (I.5 } \\
\text { T). }\end{array}$ & $\begin{array}{l}\text { Chi-square } \\
\text { test }\end{array}$ & $\begin{array}{l}69 \% \text { of joints } \\
\text { with pain had } \\
\text { JE }(p=.001) \text {. } \\
\text { OR for this } \\
\text { association was } \\
3.8 \text {. }\end{array}$ & $\begin{array}{l}\text { All } \\
\text { participants } \\
\text { were } q \text {. }\end{array}$ \\
\hline & & $\begin{array}{l}\text { All participants } \\
\text { were female. }\end{array}$ & $\begin{array}{l}\text { Examiners had } \\
\text { acceptable levels of } \\
\text { reliability. }\end{array}$ & $\begin{array}{l}\text { JE defined: area } \\
\text { of high signal } \\
\text { intensity area } \\
\text { within either } \\
\text { joint space in } \\
\text { open and closed } \\
\text { position. }\end{array}$ & OR & $\begin{array}{l}80 \% \text { of joints } \\
\text { with pain had } \\
\text { ID }(p=.332) \text {. } \\
\text { OR for this } \\
\text { association was } \\
\text { I.8. } \\
84 \% \text { of joints } \\
\text { with pain } \\
\text { had reported } \\
\text { experiencing } \\
\text { pain at the onset } \\
\text { of study ( }= \\
.001) \text {. OR for } \\
\text { this association } \\
\text { was } 49 .\end{array}$ & \\
\hline \multirow[t]{4}{*}{$\begin{array}{l}\text { Rudisch et } \\
\text { al. }{ }^{19}\end{array}$} & $\begin{array}{l}\text { Aim:To } \\
\text { investigate the } \\
\text { association } \\
\text { between TMJ- } \\
\text { Pain and MRI } \\
\text { findings of ID } \\
\text { and JE. }\end{array}$ & $\mathrm{N}=4 \mathrm{I} ; 82 \mathrm{TMJ}$ & $\begin{array}{l}\text { a. Study group: } \\
\text { Participants } \\
\text { underwent CDC/ } \\
\text { TMD examination. }\end{array}$ & $\begin{array}{l}\text { T2-weighted } \\
\text { sagittal } \\
\text { sequences ( } 1.5 \mathrm{~T} \text {, } \\
3 \text { mm thick cuts) }\end{array}$ & Chi-square & $\begin{array}{l}80.5 \% \text { of joints } \\
\text { with pain and } \\
46.3 \% \text { of joints } \\
\text { without pain } \\
\text { had ID. }\end{array}$ & $\begin{array}{l}\text { Reliability } \\
\text { of clinical } \\
\text { examiners and } \\
\text { radiologists } \\
\text { was not } \\
\text { described. }\end{array}$ \\
\hline & & $78 \%+, 22 \% \hat{\jmath}$. & & $\begin{array}{l}\text { JE defined: area } \\
\text { of more than } \\
\text { one high signal } \\
\text { intensity line } \\
\text { in the region } \\
\text { of joint space, } \\
\text { evident in } \\
\text { at least two } \\
\text { consecutive } \\
\text { sections. }\end{array}$ & $\begin{array}{l}\text { Kappa } \\
\text { statistical test }\end{array}$ & $\begin{array}{l}58.5 \% \text { of joints } \\
\text { with pain and } \\
26.8 \% \text { of joints } \\
\text { without pain } \\
\text { had JE. }\end{array}$ & \\
\hline & & $\begin{array}{l}\text { age: } 39.1 \text { yrs. (17-78 } \\
\text { yrs.) }\end{array}$ & & & & $\begin{array}{l}\text { ID and JE were } \\
\text { statistically } \\
\text { associated with } \\
\text { TMJ-Pain. } \\
\text { TMJ-Pain and ID } \\
\text { had a } K \text { value of } \\
0.34 \text { (poor). }\end{array}$ & \\
\hline & & & & & & $\begin{array}{l}\text { TMJ-Pain and JE } \\
\text { had a } \mathrm{K} \text { value } \\
\text { of } 0.32 \text { (poor). }\end{array}$ & \\
\hline
\end{tabular}


Table Continued

\begin{tabular}{|c|c|c|c|c|c|c|c|}
\hline $\begin{array}{l}\text { Author } \\
\text { and Year }\end{array}$ & Study aim & Demographics & $\begin{array}{l}\text { TMJ pain } \\
\text { assessment } \\
\text { methodology }\end{array}$ & $\begin{array}{l}\text { Imaging } \\
\text { assessment } \\
\text { methodology }\end{array}$ & Statistics & Conclusion & Limitations \\
\hline \multirow[t]{2}{*}{$\begin{array}{l}\text { Segami et } \\
\text { al. }^{6}\end{array}$} & $\begin{array}{l}\text { Aim:To } \\
\text { characterize } \\
\text { JE with } \\
\text { arthroscopic } \\
\text { findings }\end{array}$ & $\mathrm{N}=47 ; 47 \mathrm{TMJs}$ & $\begin{array}{l}\text { Study group: } \\
\text { Participants } \\
\text { underwent }\end{array}$ & $\begin{array}{l}\text { T2-wighted } \\
\text { sagittal } \\
\text { sequences (I.5T, } \\
3 \text { mm thick } \\
\text { slices). }\end{array}$ & $\begin{array}{l}\text { Mann-Whitney } \\
\text { U test }\end{array}$ & $\begin{array}{l}68.1 \% \text { of joints } \\
\text { had JE. }\end{array}$ & $\begin{array}{l}\text { Control group } \\
\text { only had } 3 \text {. }\end{array}$ \\
\hline & & $\begin{array}{l}\text { age: } 39 \text { yrs. (I 3-76 } \\
\text { yrs.) }\end{array}$ & $\begin{array}{l}\text { 2.VAS to measure } \\
\text { pain intensity. }\end{array}$ & $\begin{array}{l}\text { JE defined: high- } \\
\text { signal area in the } \\
\text { upper and lower } \\
\text { joint spaces. }\end{array}$ & $\begin{array}{l}\text { Spearman's } \\
\text { correlation } \\
\text { co-efficient }\end{array}$ & $\begin{array}{l}\text { VAS score had } \\
\text { no statistical } \\
\text { correlation with } \\
\text { the presence } \\
\text { of JE. }\end{array}$ & $\begin{array}{l}\text { Clinical } \\
\text { examination } \\
\text { was not } \\
\text { adequately } \\
\text { described. } \\
\text { Reliability } \\
\text { of clinical } \\
\text { examiners and } \\
\text { radiologists } \\
\text { was not } \\
\text { described. } \\
\text { No } \\
\text { information } \\
\text { provided } \\
\text { regarding } \\
\text { the type of } \\
\text { treatment } \\
\text { participants } \\
\text { were involved } \\
\text { in before } \\
\text { undergoing } \\
\text { MRI. }\end{array}$ \\
\hline \multirow[t]{4}{*}{$\begin{array}{l}\text { Shaefer et } \\
\text { al. }^{7}\end{array}$} & Aim: & $\mathrm{N}=30 ; 30 \mathrm{TMJs}$ & $\begin{array}{l}\text { Study group: } \\
\text { Participants } \\
\text { underwent }\end{array}$ & $\begin{array}{l}\text { T2-weighted } \\
\text { sagittal } \\
\text { sequences }\end{array}$ & $\begin{array}{l}\text { Sensitivity and } \\
\text { Specificity }\end{array}$ & $\begin{array}{l}85 \% \text { of subjects } \\
\text { with TMJ } \\
\text { arthralgia had JE. }\end{array}$ & $\begin{array}{l}\text { All subjects } \\
\text { were }+ \text {. }\end{array}$ \\
\hline & $\begin{array}{l}\text { a. To evaluate } \\
\text { the association } \\
\text { between TMJ } \\
\text { arthralgia and JE. }\end{array}$ & $100 \% q$ & $\begin{array}{l}\text { a. RDC/TMD } \\
\text { examination, } \\
\text { Modified SSI } \\
\text { for severity of } \\
\text { symptoms. }\end{array}$ & $\begin{array}{l}\text { JE defined: } \\
\text { presence of any } \\
\text { high intensity } \\
\text { signal within } \\
\text { either joint } \\
\text { space. }\end{array}$ & T-test & $\begin{array}{l}72 \% \text { of subjects } \\
\text { without any TMJ } \\
\text { pain had JE. }\end{array}$ & $\begin{array}{l}\text { The criterion } \\
\text { for TMJ } \\
\text { arthralgia was } \\
\text { not consistent } \\
\text { with RDC/ } \\
\text { TMD } \\
\text { specifications. }\end{array}$ \\
\hline & $\begin{array}{l}\text { b. To evaluate } \\
\text { the validity } \\
\text { of I pound } \\
\text { of palpating } \\
\text { pressure for } \\
\text { diagnosing } \\
\text { arthralgia. }\end{array}$ & $\begin{array}{l}\text { age of study group: } \\
31.3 \pm 9 \text { yrs. }\end{array}$ & $\begin{array}{l}\text { b. Examiners were } \\
\text { calibrated. }\end{array}$ & $\begin{array}{l}\text { Reliability of the } \\
\text { radiologist to } \\
\text { determine JE was } \\
\mathrm{K}=0.85 \text {. }\end{array}$ & Paired t-test & $\begin{array}{l}\text { MRI effusion } \\
\text { sensitivity } \\
\text { was } 85 \% \text {, and } \\
\text { specificity was } \\
28 \% \text {. }\end{array}$ & $\begin{array}{l}\text { Use of the } \\
\text { most painful } \\
\text { joint instead } \\
\text { of both or an } \\
\text { average. }\end{array}$ \\
\hline & & $\begin{array}{l}\text { age of control } \\
\text { group: } 31.6 \pm 6 \text { yrs. }\end{array}$ & & & & $\begin{array}{l}\text { Sensitivity } \\
\text { and specificity } \\
\text { of I pound } \\
\text { of palpating } \\
\text { pressure was } \\
27 \% \text { and } 100 \% \text {, } \\
\text { respectively. }\end{array}$ & \\
\hline
\end{tabular}


Table Continued...

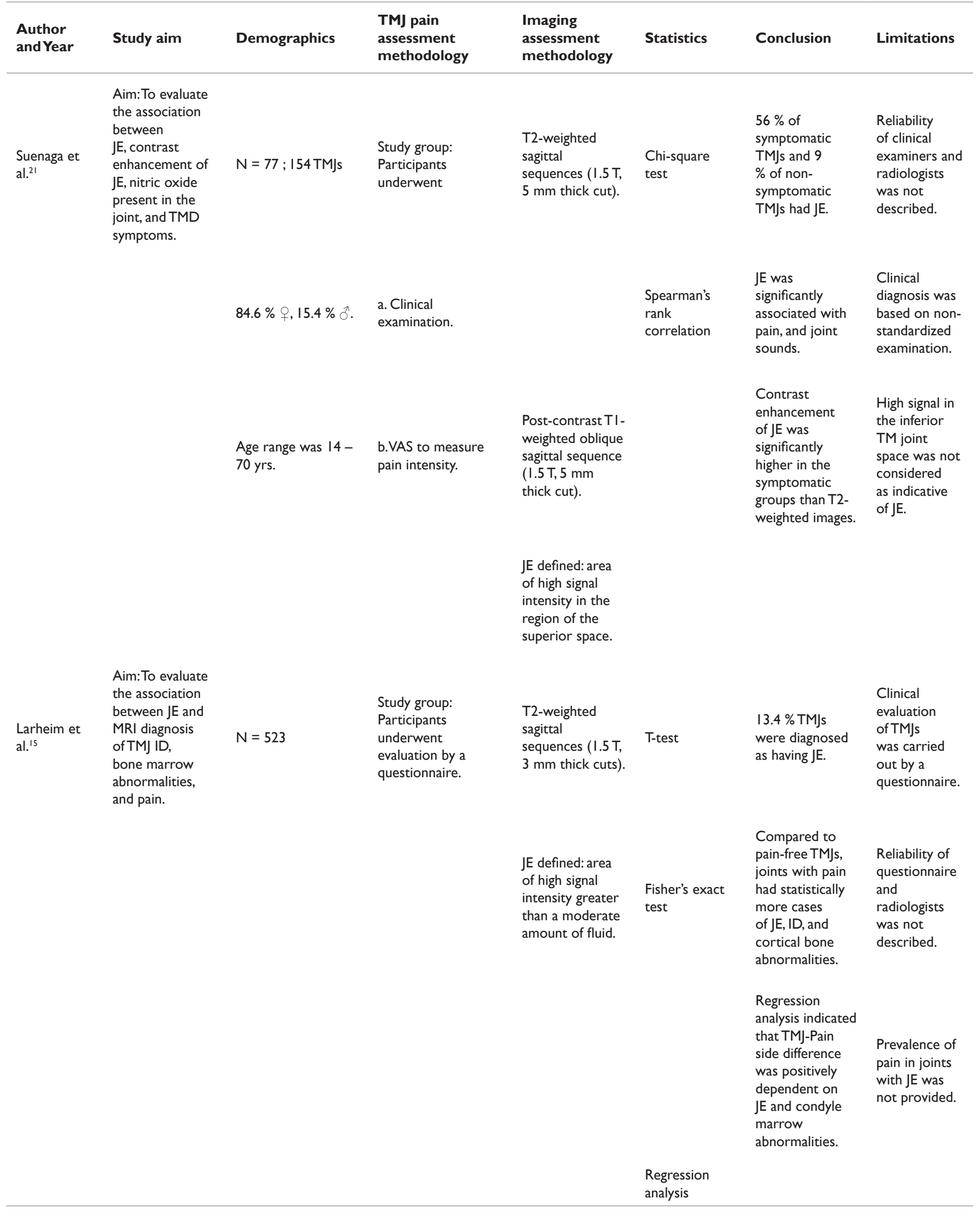


Table Continued.

\begin{tabular}{|c|c|c|c|c|c|c|c|}
\hline $\begin{array}{l}\text { Author } \\
\text { and Year }\end{array}$ & Study aim & Demographics & $\begin{array}{l}\text { TMJ pain } \\
\text { assessment } \\
\text { methodology }\end{array}$ & $\begin{array}{l}\text { Imaging } \\
\text { assessment } \\
\text { methodology }\end{array}$ & Statistics & Conclusion & Limitations \\
\hline \multirow[t]{4}{*}{$\begin{array}{l}\text { Takahashi et } \\
\text { al. }^{22}\end{array}$} & $\begin{array}{l}\text { Aim: To } \\
\text { investigate the } \\
\text { association } \\
\text { between JE, joint } \\
\text { pain, and protein } \\
\text { levels in joint } \\
\text { lavage fluid of } \\
\text { patients with ID } \\
\text { and OA. }\end{array}$ & $\mathrm{N}=26 ; 38 \mathrm{TMJs}$ & $\begin{array}{l}\text { Study group: } \\
\text { Participants } \\
\text { underwent clinical } \\
\text { examination. }\end{array}$ & $\begin{array}{l}\text { T2-weighted } \\
\text { sagittal } \\
\text { sequences ( } 1.5 \mathrm{~T} \text {, } \\
5 \mathrm{~mm} \text { thick cut). }\end{array}$ & $\begin{array}{l}\text { Fisher's exact } \\
\text { test }\end{array}$ & $\begin{array}{l}80 \% \text { of TMJs } \\
\text { with pain and } \\
38.5 \% \text { of pain- } \\
\text { free TMJs had JE } \\
(p<.05) .\end{array}$ & $\begin{array}{l}\text { Reliability } \\
\text { of clinical } \\
\text { examiners and } \\
\text { radiologists } \\
\text { was not } \\
\text { described. }\end{array}$ \\
\hline & & $84.2 \%+, 15.8 \%$ ठ & & $\begin{array}{l}\text { JE defined: area } \\
\text { of high signal } \\
\text { intensity in the } \\
\text { region of the } \\
\text { superior or } \\
\text { inferior joint } \\
\text { space. }\end{array}$ & Student's t-test & $\begin{array}{l}\text { Total protein } \\
\text { concentration } \\
\text { was statistically } \\
\text { higher in the } \\
\text { group without } \\
\text { TM joint pain } \\
\text { than in the } \\
\text { group with pain. }\end{array}$ & $\begin{array}{l}\text { Clinical } \\
\text { examination } \\
\text { was not } \\
\text { adequately } \\
\text { described. }\end{array}$ \\
\hline & & $\begin{array}{l}\text { age: } 43.2 \text { yrs. ( } 16- \\
67 \text { yrs.) }\end{array}$ & & & & & $\begin{array}{l}\text { High signal in } \\
\text { the inferior } \\
\text { TM joint } \\
\text { space was not } \\
\text { considered } \\
\text { as indicative } \\
\text { of JE. }\end{array}$ \\
\hline & & & & & & & $\begin{array}{l}\text { Definition of } \\
\text { the control } \\
\text { group was not } \\
\text { clear. }\end{array}$ \\
\hline \multirow[t]{3}{*}{$\begin{array}{l}\text { Adame et } \\
\text { al. }^{27}\end{array}$} & $\begin{array}{l}\text { Aim: To correlate } \\
\text { JE with clinical } \\
\text { and MRI findings. }\end{array}$ & $\mathrm{N}=142 ; 169 \mathrm{TM} \mathrm{Js}$ & $\begin{array}{l}\text { Study group: } \\
\text { Participants } \\
\text { underwent clinical } \\
\text { examination. }\end{array}$ & $\begin{array}{l}\text { T2-wighted } \\
\text { sagittal } \\
\text { sequences (0.5T, } \\
5 \text { mm thick } \\
\text { slices). }\end{array}$ & $\begin{array}{l}\text { Pearson's Chi- } \\
\text { square test }\end{array}$ & $\begin{array}{l}65 \% \text { of } \\
\text { participants with } \\
\text { JE had TM joint } \\
\text { pain. }\end{array}$ & $\begin{array}{l}\text { Clinical } \\
\text { examination } \\
\text { was not } \\
\text { adequately } \\
\text { described. }\end{array}$ \\
\hline & & $88.9 \%+$, II.I \% ठ & & $\begin{array}{l}\text { JE defined: hyper- } \\
\text { intensity signal } \\
\text { in either joint } \\
\text { space, seen in } \\
\text { two consecutive } \\
\text { image cuts. }\end{array}$ & & $\begin{array}{l}69.6 \% \text { of } \\
\text { participants } \\
\text { without JE had } \\
\text { TM joint pain. }\end{array}$ & $\begin{array}{l}\text { Reliability } \\
\text { of clinical } \\
\text { examiners and } \\
\text { radiologists } \\
\text { was not } \\
\text { described. }\end{array}$ \\
\hline & & $\begin{array}{l}\text { age: } 25.4 \text { yrs. (14-74 } \\
\text { yrs.) }\end{array}$ & & & & $\begin{array}{l}\text { TMJs with } \\
\text { effusion had } \\
\text { significantly } \\
\text { lower incidence } \\
\text { of TM joint } \\
\text { clicking than } \\
\text { control group. }\end{array}$ & $\begin{array}{l}\text { Data } \\
\text { presented in } \\
\text { table does } \\
\text { not match the } \\
\text { text. }\end{array}$ \\
\hline \multirow[t]{2}{*}{$\begin{array}{l}\text { Murakami et } \\
\text { al. }{ }^{17}\end{array}$} & $\begin{array}{l}\text { Aim: To } \\
\text { investigate the } \\
\text { association } \\
\text { between TMJ } \\
\text { pain levels and JE. }\end{array}$ & $N=19 ; 19$ joints & $\begin{array}{l}\text { Study group: } \\
\text { Participants } \\
\text { underwent }\end{array}$ & $\begin{array}{l}\text { T2-weighted } \\
\text { sagittal } \\
\text { sequences (I.5 } \\
\text { T). }\end{array}$ & Paired t-test & $\begin{array}{l}52.6 \% \text { of } \\
\text { participants } \\
\text { had JE. }\end{array}$ & $\begin{array}{l}\text { All } \\
\text { participants } \\
\text { were } q \text {. }\end{array}$ \\
\hline & & $\begin{array}{l}\text { All participants } \\
\text { were } q \text { and were } \\
\text { diagnosed as having } \\
\text { disk displacement } \\
\text { without reduction. }\end{array}$ & $\begin{array}{l}\text { a. Clinical } \\
\text { examination. }\end{array}$ & $\begin{array}{l}\text { JE defined: area } \\
\text { of more than } \\
\text { a line of high } \\
\text { signal intensity } \\
\text { in the region } \\
\text { of superior or } \\
\text { inferior joint } \\
\text { space. }\end{array}$ & & $\begin{array}{l}\text { No statistically } \\
\text { significant } \\
\text { difference } \\
\text { was observed } \\
\text { between the } \\
\text { groups for VAS } \\
\text { pain score, pain } \\
\text { questionnaire } \\
\text { scores, and total } \\
\text { pain score. }\end{array}$ & $\begin{array}{l}\text { Clinical } \\
\text { examination } \\
\text { was not } \\
\text { adequately } \\
\text { described. }\end{array}$ \\
\hline
\end{tabular}


Table Continued..

\begin{tabular}{|c|c|c|c|c|c|c|c|}
\hline $\begin{array}{l}\text { Author } \\
\text { and Year }\end{array}$ & Study aim & Demographics & $\begin{array}{l}\text { TMJ pain } \\
\text { assessment } \\
\text { methodology }\end{array}$ & $\begin{array}{l}\text { Imaging } \\
\text { assessment } \\
\text { methodology }\end{array}$ & Statistics & Conclusion & Limitations \\
\hline \multirow{6}{*}{$\begin{array}{l}\text { Westesson } \\
\text { et al. }{ }^{24}\end{array}$} & \multirow{6}{*}{$\begin{array}{l}\text { Aim:To } \\
\text { investigate the } \\
\text { association } \\
\text { between JE and } \\
\text { clinical symptoms } \\
\text { of TMJ pain, and } \\
\text { MRI diagnosis of } \\
\text { ID and OA. }\end{array}$} & $\begin{array}{l}\text { age: } 39.1 \text { yrs. (|4-6| } \\
\text { yrs.) }\end{array}$ & $\begin{array}{l}\text { b.VAS and Pain level } \\
\text { questionnaire to } \\
\text { measure intensity } \\
\text { of pain. }\end{array}$ & & \multirow{6}{*}{$\begin{array}{l}\text { No report } \\
\text { on statistical } \\
\text { analysis. }\end{array}$} & & $\begin{array}{l}\text { Reliability } \\
\text { of clinical } \\
\text { examiners and } \\
\text { radiologists } \\
\text { was not } \\
\text { described }\end{array}$ \\
\hline & & $\mathrm{N}=390 ; 780 \mathrm{TMJs}$ & $\begin{array}{l}\text { Study group: } \\
\text { Participants had } \\
\text { TMD pain and } \\
\text { dysfunction. }\end{array}$ & $\begin{array}{l}\text { T2-weighted } \\
\text { sagittal } \\
\text { sequences (I.5 } \\
\text { T). }\end{array}$ & & $\begin{array}{l}7 \% \text { of joints } \\
\text { with normal disk } \\
\text { position had JE. }\end{array}$ & $\begin{array}{l}\text { No } \\
\text { demographic } \\
\text { details } \\
\text { provided. }\end{array}$ \\
\hline & & & $\begin{array}{l}\text { Control group: } \\
\text { Participants did not } \\
\text { have any TMD pain } \\
\text { or dysfunction. }\end{array}$ & $\begin{array}{l}\text { JE defined: } \\
\text { more than a } \\
\text { line of high } \\
\text { signal intensity } \\
\text { in the region } \\
\text { of superior or } \\
\text { inferior joint } \\
\text { space. }\end{array}$ & & $\begin{array}{l}40 \% \text { of joints } \\
\text { with DDwR } \\
\text { had JE. }\end{array}$ & $\begin{array}{l}\text { Clinical } \\
\text { examination } \\
\text { was not } \\
\text { adequately } \\
\text { described. }\end{array}$ \\
\hline & & & & & & $\begin{array}{l}50 \% \text { of joints } \\
\text { with DDwoR } \\
\text { had JE. }\end{array}$ & $\begin{array}{l}\text { Details for } \\
\text { blindness of } \\
\text { the radiologist } \\
\text { not provided. }\end{array}$ \\
\hline & & & & & & $\begin{array}{l}27 \% \text { of joints } \\
\text { with OA had JE. }\end{array}$ & $\begin{array}{l}\text { Reliability } \\
\text { of clinical } \\
\text { examiners and } \\
\text { radiologists } \\
\text { was not } \\
\text { described. }\end{array}$ \\
\hline & & & & & & $\begin{array}{l}\text { Statistically } \\
\text { significant } \\
\text { association was } \\
\text { present between } \\
\text { JE and joints } \\
\text { with severe pain. }\end{array}$ & \\
\hline
\end{tabular}

\section{Discussion}

A systematic review was conducted to determine if there is an association between joint effusion, self-reported TMJ pain and arthralgia of the TMJ. Based on the review of 32 articles published on this topic, the body of literature was unable to provide evidence to support or refute the association between joint effusion and selfreported pain TMJ pain during examination or between joint effusion and arthralgia, as defined by RDC-TMD. Systematic review of the studies indicated that the majority of investigations had reported a statistically significant association between joint effusion and TMJ self-reported pain during examination and joint effusion and arthralgia as defined by RDC-TMD. However, these results need to be interpreted with caution due to the presence of several crucial methodological limitations. Some of these studies failed to apply a standardized clinical examination. ${ }^{4-36}$ This may result in failure to identify the true source of pain associated with TMD, or may result in using indeterminate classifications, such as the inclusion of participants with "tenderness" to palpation instead of "pain", or inclusion of participants with pain of muscular origin rather than of the TMJ. Furthermore, some investigations failed to report the reliability of the clinical examiners and radiologists. ${ }^{10-26}$ Reliability is the overall consistency of a measure and is considered an important factor in identification of TMD and MRI-based findings since the prevalence of both conditions in the normal population range relatively low, ${ }^{31}$ and overlooking reliability may influence the results in favour of a false positive association and inconsistent reporting. Another reason for disparity in reporting may be due to poor consensus among the authors for the operational definition of joint effusion. Some of the investigators defined effusion as presence of a high intensity signal on T2 images only in the superior joint space, while other considered presence of a high intensity signal in the superior or inferior joint space as indicative of joint effusion. Similarly, some authors quantified the joint effusion into multiple relative categories. However, none of these investigations reported the reliability and validity of radiologist to quantify effusion, which limits 
the utility of this measure. Nonetheless, among the studies reporting quantification of effusion, the results were not consistent.

\section{Conclusion}

In summary, based on the findings of this systematic review, the association between MRI diagnosis of joint effusion and self-reported TMJ-pain as well as between joint effusion and arthralgia, as defined by RDC-TMD, was not determined. This may be attributed to several methodological limitations, and heterogeneity in the operational definition of joint effusion. Investigations using validated and reliable clinical and radiologic criteria with appropriate case and control groups that have been adequately characterized are required. This will help determine, if any, association is present between MRI diagnosis of joint effusion and joint pain, and joint effusion and arthralgia.

\section{Funding}

None.

\section{Acknowledgements}

None.

\section{Conflicts of interest}

The authors declare no potential conflicts of interest with respect to the authorship and/or publication of this article.

\section{References}

1. Greene CS. Diagnosis and treatment of temporomandibular disorders: emergence of a new care guidelines statement. Oral Surg Oral Med Oral Pathol Oral Radiol Endod. 2010;110(2):137-139.

2. Dworkin SF, LeResche L. Research diagnostic criteria for temporomandibular disorders: review, criteria, examinations and specifications, critique. J Craniomandib Disord. 1992;6(4):301-355.

3. Ahmad M, Hollender L, Anderson Q, et al. Research diagnostic criteria for temporomandibular disorders (RDC/TMD): development of image analysis criteria and examiner reliability for image analysis. Oral Surg Oral Med Oral Pathol Oral Radiol Endod. 2009;107(6):844-860.

4. Oliveira JX, Rosa JA, Dutra ME, et al. Assessing joint effusion and bone changes of the head of the mandible in MR images of symptomatic patients. Braz Oral Res. 2013;27(1):37-41.

5. Limchaichana N, Nilsson H, Ekberg EC, et al. Clinical diagnoses and MRI findings in patients with TMD pain. J Oral Rehabil. 2007;34(4):237-245.

6. Segami N, Nishimura M, Kaneyama K, et al. Does joint effusion on T2 magnetic resonance images reflect synovitis? Comparison of arthroscopic findings in internal derangements of the temporomandibular joint. Oral Surg Oral Med Oral Pathol Oral Radiol Endod. 2001;92(3):341-345.

7. Shaefer JR, Jackson DL, Schiffman EL, et al. Pressure-pain thresholds and MRI effusions in TMJ arthralgia. J Dent Res. 2001;80(10):1935-1939.

8. Khawaja SN, Crow H, Mahmoud RF, et al. Is There an Association Between Temporomandibular Joint Effusion and Arthralgia? J Oral Maxillofac Surg. 2017;75(2):268-275.

9. Bas B, Yilmaz N, Gokce E, et al. Ultrasound assessment of increased capsular width in temporomandibular joint internal derangements: relationship with joint pain and magnetic resonance grading of joint effusion. Oral Surg Oral Med Oral Pathol Oral Radiol Endod. 2011;112(1):112-117.

10. Costa AL, D’Abreu A, Cendes F. Temporomandibular joint internal derangement: association with headache, joint effusion, bruxism, and joint pain. J Contemp Dent Pract. 2008;9(6):9-16.
11. Guler N, Yatmaz PI, Ataoglu H, et al. Temporomandibular internal derangement: correlation of MRI findings with clinical symptoms of pain and joint sounds in patients with bruxing behaviour. Dentomaxillofac Radiol. 2003;32(5):304-310.

12. Haley DP, Schiffman EL, Lindgren BR, et al. The relationship between clinical and MRI findings in patients with unilateral temporomandibular joint pain. J Am Dent Assoc. 2001;132(4):476-481.

13. Kaneyama K, Segami N, Yoshimura H, et al. Increased levels of soluble cytokine receptors in the synovial fluid of temporomandibular joint disorders in relation to joint effusion on magnetic resonance images. $J$ Oral Maxillofac Surg. 2010;68(5):1088-1093.

14. Lamot U, Strojan P, Surlan Popovic K. Magnetic resonance imaging of temporomandibular joint dysfunction-correlation with clinical symptoms, age, and gender. Oral Surg Oral Med Oral Pathol Oral Radiol. 2013;116(2):258-263.

15. Larheim TA, Westesson PL, Sano T. MR grading of temporomandibular joint fluid: association with disk displacement categories, condyle marrow abnormalities and pain. Int J Oral Maxillofac Surg. 2001;30(2):104-112.

16. Manfredini D, Tognini F, Zampa V, et al. Predictive value of clinical findings for temporomandibular joint effusion. Oral Surg Oral Med Oral Pathol Oral Radiol Endod. 2003;96(5):521-526.

17. Murakami K, Nishida M, Bessho K, et al. MRI evidence of high signal intensity and temporomandibular arthralgia and relating pain. Does the high signal correlate to the pain? Br J Oral Maxillofac Surg. 1996;34(3):220-224.

18. Park JW, Song HH, Roh HS, et al. Correlation between clinical diagnosis based on RDC/TMD and MRI findings of TMJ internal derangement. Int J Oral Maxillofac Surg. 2012;41(1):103-108.

19. Rudisch A, Innerhofer K, Bertram S, et al. Magnetic resonance imaging findings of internal derangement and effusion in patients with unilateral temporomandibular joint pain. Oral Surg Oral Med Oral Pathol Oral Radiol Endod. 2001;92(5):566-571.

20. Santos KC, Dutra ME, Warmling LV, et al. Correlation among the changes observed in temporomandibular joint internal derangements assessed by magnetic resonance in symptomatic patients. J Oral Maxillofac Surg. 2013;71(9):1504-1512.

21. Suenaga S, Abeyama K, Hamasaki A, et al. Temporomandibular disorders: relationship between joint pain and effusion and nitric oxide concentration in the joint fluid. Dentomaxillofac Radiol. 2001;30(4):214-218.

22. Takahashi T, Nagai H, Seki H, et al. Relationship between joint effusion, joint pain, and protein levels in joint lavage fluid of patients with internal derangement and osteoarthritis of the temporomandibular joint. J Oral Maxillofac Surg. 1999;57(10):1187-1193; discussion 1193-1184.

23. Tanaka T, Morimoto Y, Masumi S, et al. Utility of frequency-selective fat saturation T2-weighted MR images for the detection of joint effusion in the temporomandibular joint. Dentomaxillofac Radiol. 2002;31(5):305-312.

24. Westesson PL, Brooks SL. Temporomandibular joint: relationship between MR evidence of effusion and the presence of pain and disk displacement. AJR Am J Roentgenol. 1992;159(3):559-563.

25. Yamamoto M, Sano T, Okano T. Magnetic resonance evidence of joint fluid with temporomandibular joint disorders. J Comput Assist Tomogr. 2003;27(5):694-698.

26. Yano K, Sano T, Okano T. A longitudinal study of magnetic resonance (MR) evidence of temporomandibular joint (TMJ) fluid in patients with TMJ disorders. Cranio. 2004;22(1):64-71.

27. Adame CG, Monje F, Offnoz M, et al. Effusion in magnetic resonance imaging of the temporomandibular joint: a study of 123 joints. J Oral Maxillofac Surg. 1998;56(3):314-318. 
28. Farina D, Bodin C, Gandolfi S, et al. TMJ disorders and pain: assessmen by contrast-enhanced MRI. Eur J Radiol. 2009;70(1):25-30.

29. Guler N, Uckan S, Imirzalioglu P, et al. Temporomandibular joint internal derangement: relationship between joint pain and MR grading of effusion and total protein concentration in the joint fluid. Dentomaxillofac Radiol. 2005;34(3):175-181.

30. Segami N, Miyamaru M, Nishimura M, et al. Does joint effusion on T2 magnetic resonance images reflect synovitis? Part 2. Comparison of concentration levels of proinflammatory cytokines and total protein in synovial fluid of the temporomandibular joint with internal derangements and osteoarthrosis. Oral Surg Oral Med Oral Pathol Oral Radiol Endod. 2002;94(4):515-521.

31. Schiffman E, Ohrbach R, Truelove E, et al. Diagnostic Criteria for Temporomandibular Disorders (DC/TMD) for Clinical and Research Applications: Recommendations of the International RDC/TMD Consortium Network* and Orofacial Pain Special Interest Groupdagger. $J$ Oral Facial Pain Headache. 2014;28(1):6-27.

32. Nakaoka K, Hamada Y, Holmlund AB, et al. The changes of joint effusion on MRI and arthroscopic findings after visually guided TMJ irrigation correlated to the clinical outcome. Oral Surg Oral Med Oral Pathol Oral Radiol Endod. 2009;108(1):99-104.
33. Emshoff R, Brandlmaier I, Gerhard S, et al. Magnetic resonance imaging predictors of temporomandibular joint pain. $J$ Am Dent Assoc. 2003;134(6):705-714

34. Emshoff R, Brandlmaier I, Bertram S, et al. Risk factors for temporomandibular joint pain in patients with disc displacement without reduction - a magnetic resonance imaging study. J Oral Rehabil. 2003;30(5):537-543.

35. Emshoff R, Brandlmaier I, Bertram S, et al. Relative odds of temporomandibular joint pain as a function of magnetic resonance imaging findings of internal derangement, osteoarthrosis, effusion, and bone marrow edema. Oral Surg Oral Med Oral Pathol Oral Radiol Endod. 2003;95(4):437-445.

36. Emshoff R, Brandimaier I, Bertram S, et al. Magnetic resonance imaging findings of osteoarthrosis and effusion in patients with unilateral temporomandibular joint pain. Int J Oral Maxillofac Surg. 2002;31(6):598-602. 